\title{
A More General MSSM Parameter Scan
}

Here a more general MSSM parameter scan than that described in section 8.3 is considered, following [1],2]. In addition, while the benchmark results given there used the Renormalisation Group Equation (RGE) approach [3] with recent modifications bringing them into agreement with the Full Diagrammatic Calculations (FDC) [ [1], here the FDC are used directly.

The parameters shown in Table 11 are the input parameters for the calculations of the physical Higgs, sfermion, chargino, and neutralino masses and production rates, and they are varied in the ranges shown. The $A$ mass is scanned in steps of $1 \mathrm{GeV} / \mathrm{c}^{2}$ up to $200 \mathrm{GeV} / \mathrm{c}^{2}$ and in larger steps thereafter. For each $m_{\mathrm{A}}, 2700$ parameter combinations are investigated. The range of $\tan \beta$ is restricted to $0.5 \leq \tan \beta \leq 50$ : the lower bound is described for example in [6], and affects the theoretically allowed regions in the $\left(m_{\mathrm{h}}, m_{\mathrm{A}}\right)$ plane; varying the upper bound has no significant effect on the results. The range of $\mu$ is restricted to $\pm 500 \mathrm{GeV} / \mathrm{c}^{2}$, since very large values are disfavored by Charge- and ColorBreaking (CCB) global minima in the MSSM [5]. Finally, in the RGE approach used for the benchmark scan, $X_{t}=\sqrt{6} M_{S}$ defined the so-called $m_{\mathrm{h}}^{\max }$ scenario, and $X_{t}=0$ the no mixing scenario. In the FDC used here, the maximum scalar Higgs mass is obtained for $A=2 M_{S}$, where $X_{t}^{F D C}=A-\mu / \tan \beta$. Compared to previous similar studies [1, 2], the range of $A$ is extended from $\pm 1 M_{S}$ to $\pm 2 M_{S}$ in order to include the maximal Higgs boson mass in the scan. The top quark mass is fixed at $m_{\mathrm{t}}=175 \mathrm{GeV} / \mathrm{c}^{2}$. A value of $m_{\mathrm{t}}=180 \mathrm{GeV} / \mathrm{c}^{2}$ would increase the calculated $m_{\mathrm{h}}$ values by $2 \mathrm{GeV} / \mathrm{c}^{2}$ for large $\tan \beta$ and by $4 \mathrm{GeV} / \mathrm{c}^{2}$ for small $\tan \beta$.

\begin{tabular}{|c|c|c|c|c|c|c|}
\hline Parameter & $m_{\mathrm{A}}$ & $\tan \beta$ & $M_{S}$ & $M_{2}$ & $\mu$ & $A$ or $X_{t}$ \\
\hline this scan & $20-1000$ & $0.5-50$ & $200-1000$ & $200-1000$ & $-500-+500$ & $-2 M_{S}-+2 M_{S}$ \\
benchmark & $20-1000$ & $0.5-50$ & 1000 & 200 & -200 & $0, \sqrt{6} M_{S}$ \\
\hline
\end{tabular}

Table 1: Ranges of SUSY parameters at the electroweak scale used for independent variation in the more general MSSM parameter scan compared with those used in the benchmark scan of section 8.3. All masses are given in $\mathrm{GeV} / \mathrm{c}^{2}$.

For some parameter combinations the branching ratio into a pair of neutralinos is dominant. In such a case no limit can be derived using the channels studied in this paper. Therefore, limits from the DELPHI search for invisible decays of neutral Higgs bosons [12 are also used.

The following constraints have also been considered:

- The rate of the flavour changing neutral current process $b \rightarrow s \gamma[7]$. In the SM only amplitudes with virtual $\mathrm{W}^{ \pm}$exchange contribute, while in the MSSM there are additional contributions from supersymmetric particles and Higgs bosons $8 \|^{2}$.

- The electroweak parameter $\Delta \rho=\alpha_{\mathrm{em}} T_{\mathrm{MSSM}}$ [9]. The MSSM contributions are constrained by experiment: $T_{\mathrm{MSSM}}<0.08$ [10].

- Chargino and neutralino mass limits from direct searches [11.

But these constraints have little influence on the excluded parameter regions with the present Higgs mass limits. Therefore, the excluded parameter sets are determined from the Higgs boson searches alone.

Figures 11 to 3 present the results in the $\left(m_{\mathrm{h}}, m_{\mathrm{A}}\right),\left(m_{\mathrm{h}}, \tan \beta\right)$, and $\left(m_{\mathrm{A}}, \tan \beta\right)$ planes respectively. A given $\left(m_{\mathrm{h}}, m_{\mathrm{A}}\right)$ combination, for example, is excluded if all contrubuting

${ }^{\dagger}$ These calculations changed the expected $\mathrm{b} \rightarrow \mathrm{s} \gamma$ rates significantly. However, not all contributing terms are included and resulting assumptions are not valid in this more general scan. 
SUSY parameter sets in the ranges defined in Table — are excluded at more than 95\% confidence level after combining all search channels using the likelihood ratio method [13. The figures show three regions:

- the $95 \%$ CL excluded region (light grey),

- the theoretically not allowed region (dark).

- the allowed region (white),

and Fig. 1 also shows the region excluded by the benchmark scan (dotted line): the region excluded by the more general scan is smaller. In particular, the benchmark limits of $82.6 \mathrm{GeV} / \mathrm{c}^{2}$ on the $\mathrm{h}$ mass and $84.1 \mathrm{GeV} / \mathrm{c}^{2}$ on the A mass are reduced to $75 \mathrm{GeV} / \mathrm{c}^{2}$ and $78 \mathrm{GeV} / \mathrm{c}^{2}$, respectively. Comparison of the FDC cross sections used here and the RGE calculations used for the benchmark scan confirms that this is due to the reduced production cross-sections allowed by the extended parameter range, not due to differences between the FDC and RGE calculations. As illustrated in Table 2, low unexcluded $m_{\mathrm{h}}$ values are typically obtained for large mixing in the stop sector (large $A$, large $|\mu|$ ). In conclusion, the scan over a larger parameter region reduces the mass limits given in sections 8.3 and 9 by $8 \mathrm{GeV} / \mathrm{c}^{2}$ for the scalar and by $6 \mathrm{GeV} / \mathrm{c}^{2}$ for the pseudoscalar Higgs boson.

\begin{tabular}{|c|c|c|c|c|c|c|c|c|c|c|c|}
\hline$m_{\mathrm{A}}$ & $m_{\mathrm{h}}$ & $\tan \beta$ & $M_{S}$ & $M_{2}$ & $\mu$ & $A / M_{S}$ & $X_{t}^{F D C}$ & $m_{\tilde{\mathrm{t}} 1}$ & $m_{\tilde{\mathrm{t}} 2}$ & $\sigma_{\mathrm{hZ}}^{189}$ & $\sigma_{\mathrm{hA}}^{189}$ \\
\hline 80 & 78 & 8 & 1000 & 200 & 500 & 2 & 1938 & 979 & 1048 & 0.07 & 0.09 \\
85 & 80 & 12 & 200 & 1000 & -500 & 1 & 242 & 162 & 333 & 0.00 & 0.06 \\
85 & 86 & 10 & 1000 & 1000 & 500 & 2 & 1950 & 824 & 1174 & 0.03 & 0.04 \\
90 & 86 & 4.4 & 1000 & 200 & -100 & -2 & -1977 & 824 & 1174 & 0.11 & 0.02 \\
\hline
\end{tabular}

Table 2: Examples of unexcluded parameter combinations in the more general MSSM scan. Cross-sections for Higgs boson bremsstrahlung and pair-production are given for $\sqrt{s}=189 \mathrm{GeV}$. All masses are given in $\mathrm{GeV} / c^{2}$ and cross-sections in pb.

\section{References}

[1] J. Rosiek and A. Sopczak, Phys. Lett. 341B (1995) 419.

[2] A. Sopczak, Eur. Phys. J. C7 (1999) 107.

[3] M. Carena, M. Quiros and C. Wagner, Nucl.Phys. B461 (1996) 405.

[4] S. Heinemeyer, W. Hollik and G. Weiglein, Eur. Phys. J. C9 (1999) 343.

[5] A. Kusenko, P. Langacker and G. Segre, Phys. Rev. 54D (1996) 5824.

[6] B. Grządkowski and J.F. Gunion, Phys. Lett. 243B (1990) 301.

[7] CLEO Collaboration, CLEO CONF (98-17) (1998).

[8] M. Ciuchini, G.Degrassi, P.Gambino and G. Giudice, Nucl. Phys. 534B (1998) 3.

[9] P. Chankowski and St. Pokorski, Supersymmetric loop effects, in Perspectives on Supersymmetry, Ed. G.L.Kane, World Sci., Singapore (1997) p.27.

[10] J. Erler and D.M. Pierce, Nucl. Phys. 526B (1998) 53.

[11] DELPHI Collaboration, P. Abreu et al., Phys. Lett. B479 (2000) 129.

[12] DELPHI Collaboration, P. Abreu et al., A search for invisible Higgs bosons produced in $e^{+} e^{-}$interactions up to $\sqrt{s}=189$ GeV, CERN-EP/2000-051, submitted to Phys. Lett. B.

[13] A.L. Read, DELPHI note 97-158 PHYS 737 (1997). 


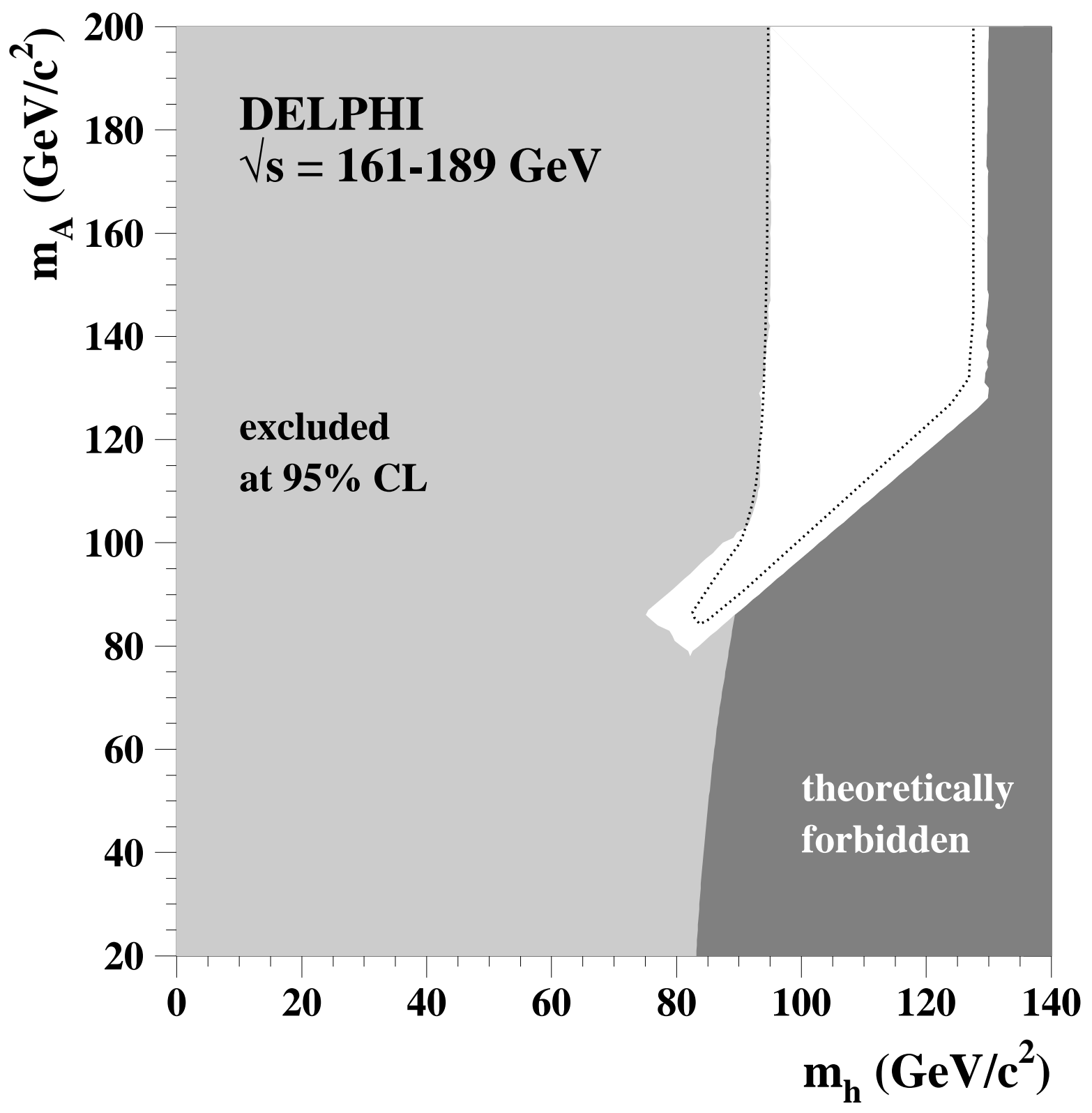

Figure 1: MSSM exclusion based on all DELPHI data from $\sqrt{s}=161 \mathrm{GeV}$ to $189 \mathrm{GeV}$ in the framework of the more general parameter scan. The region excluded at 95\% CL (light grey), the unexcluded region (white) and the theoretically forbidden region (dark grey) are shown. The dotted line marks the allowed region obtained with the benchmark scan under the assumption of maximal mixing in the stop sector. 


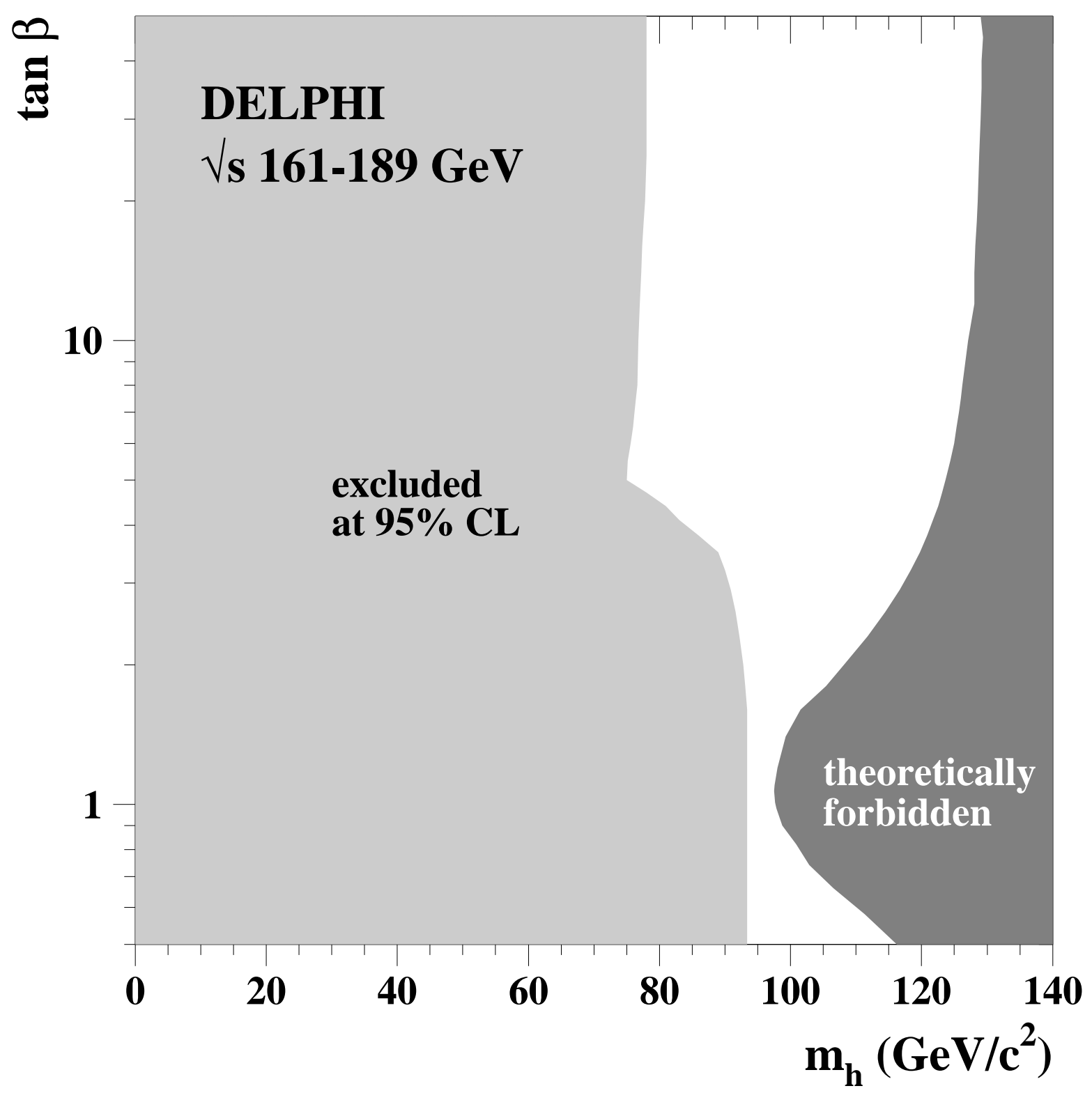

Figure 2: MSSM exclusion based on all DELPHI data from $\sqrt{s}=161 \mathrm{GeV}$ to $189 \mathrm{GeV}$ in the framework of the more general parameter scan. The region excluded at 95\% CL (light grey), the unexcluded region (white) and the theoretically forbidden region (dark grey) are shown. 


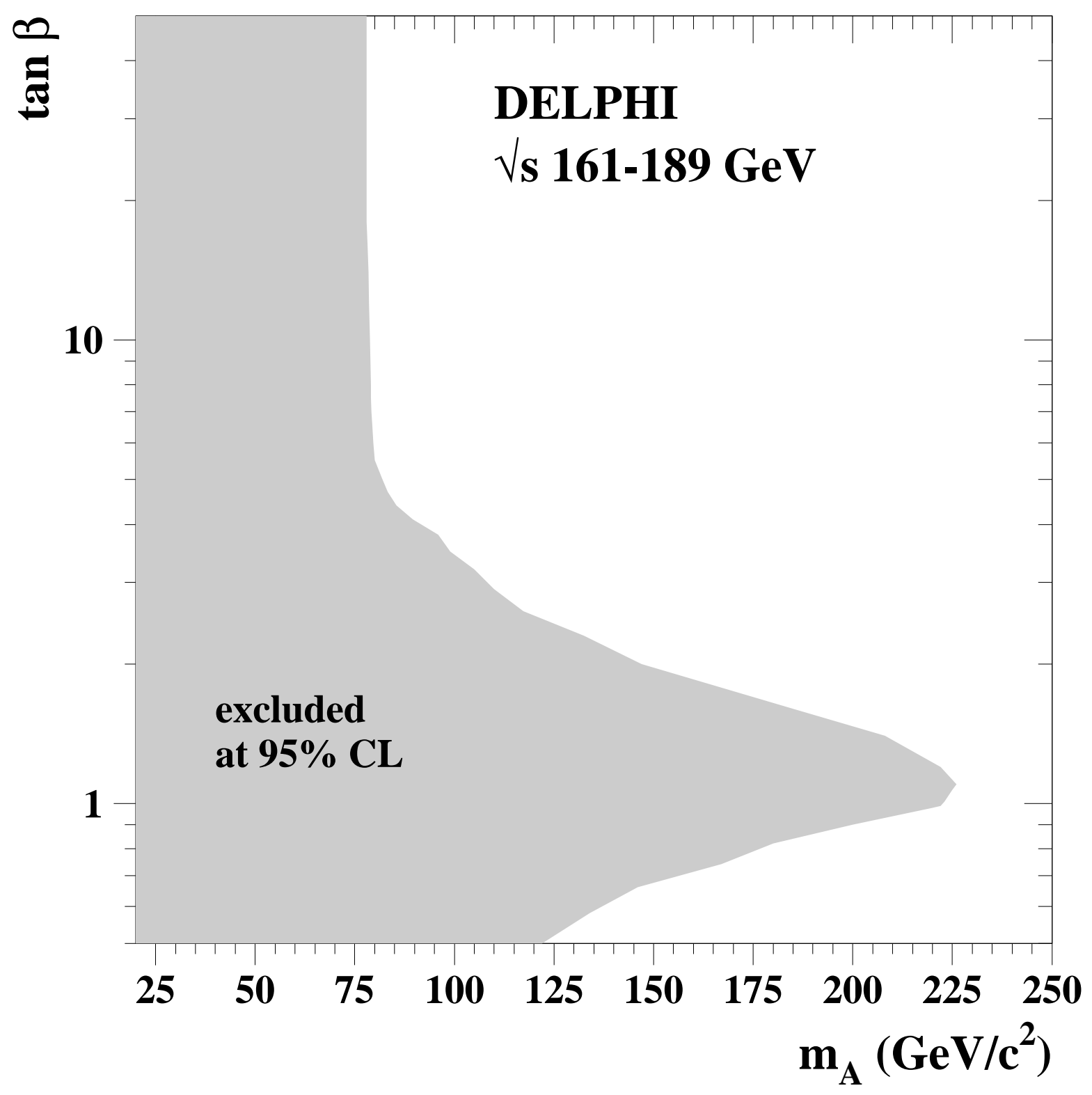

Figure 3: MSSM exclusion based on all DELPHI data from $\sqrt{s}=161 \mathrm{GeV}$ to $189 \mathrm{GeV}$ in the framework of the more general parameter scan. The region excluded at $95 \% \mathrm{CL}$ (light grey) and the unexcluded region (white) are shown. 